\title{
REVIEW
}

\section{Transcriptional regulation of hepatic stellate cell activation}

\section{A Mann, D E Smart}

Gut 2002;50:891-896

The hepatic stellate cell (HSC) is now well established as the key cellular element involved in the development of hepatic fibrosis and because of this there is considerable interest in establishing the molecular events that trigger and perpetuate HSC activation. HSC activation at the level of gene transcription requires the coordinated activity of several key transcriptional regulators of the HSC genome. The considerable advances that have been made in the past five years into the mechanisms by which specific families of transcription factors regulate the profibrogenic characteristics of the activated HSC are reviewed.

See end of article for authors' affiliations

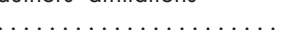

Correspondence to: Dr D A Mann, Liver Group, Division of Infection, Inflammation, and Repair, University of Southampton, Southampton General Hospital, Southampton SO16 6YD, UK: dam2@soton.ac.uk

Accepted for publication 3 July 2001
$T$ he hepatic stellate cell (HSC) is now well established as the key cellular element involved in the development of hepatic fibrosis. In response to viral, chemical, or immune insult of the liver, the normally quiescent vitamin A storing HSC undergoes a dramatic phenotypic transformation termed "activation" or "transdifferentiation". ${ }^{12}$ The process of HSC activation generates an $\alpha$ smooth muscle actin ( $\alpha$-SMA) positive myofibroblast-like cell that is proliferative and is responsible for deposition of the majority of excess extracellular matrix (ECM, predominantly types I and III collagen) that forms scar tissue in the fibrotic liver. ${ }^{1-3}$ Because of its importance in the fibrotic process, there is considerable interest in establishing the molecular events that trigger and perpetuate HSC activation.

HSC activation is a highly pleiotropic process that involves gross morphological, behavioural, and biochemical changes. ${ }^{1}$ These dramatic phenotypic changes require global reprogramming of HSC gene expression which in turn must be orchestrated by long term changes in the expression and/or activity of key transcriptional regulators of the HSC genome. Gene transcription in mammalian cells is controlled by the interaction of transcription factors with specific DNA sequences located in the regulatory regions of genes known as promoter or enhancer motifs. ${ }^{4}$ Binding of transcription factors at these sites brings them into proximity with RNA polymerase II and its associated components of the basic transcription complex that assemble in the $5^{\prime}$ untranscribed region of a gene (fig 1). The transcription factors are then able to exert either a positive or negative influence on the rate at which the basic transcription complex transcribes a gene. Because of the ability of transcription factors to exert a powerful regulatory role on the phenotype of cells, it is essential that their activity is also exquisitely controlled. The activity of a transcription factor is controlled at multiple levels. Because transcription factors are proteins, modulation of their expression and the more rapid attenuation of function via post-translational modification (phosphorylation, acetylation, ubiquitination, and interaction with coactivators and inhibitors) exert the greatest regulatory influence..$^{5-7}$ As these regulatory events are wired into a complex network of intracellular signal transduction pathways that are activated in response to cellular stimuli, they provide a finely tuned mechanism for altering transcription factor activity and subsequently the cellular phenotype according to the local requirements of a particular tissue. In the case of the injured liver the requirement is for production of the wound healing activated HSC. Perpetuation of this response in chronic liver disease results in the pathogenic state of fibrosis.

This article reviews the considerable advances that have been made in the past five years of research into the mechanisms by which specific families of transcription factors regulate the profibrogenic characteristics of the activated HSC.

\section{NFKB}

Nuclear factor $\kappa \mathrm{B}(\mathrm{NF \kappa B})$ is composed of heteroor homodimers of the Rel protein family (p65, p50, p52, c-Rel, and RelB), with the p65:p50 heterodimer being the classic NFKB complex induced by cytokine, mitogen, or UV stimulation of mammalian cells. ${ }^{89}$ The level of cellular NFKB activity is determined by the properties of its naturally occurring inhibitors, of which inhibitor of NFKB

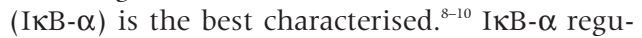
lates NFKB activity by directly interacting with the transcription factor to form inactive complexes that are localised to the cytoplasm. Stimulation of cells leads to activation of a cascade of signal transduction events that culminate in proteasome mediated degradation of I $\kappa \mathrm{B}-\alpha$ and the release of active $\mathrm{Nf \kappa B} .{ }^{10} \mathrm{NF \kappa B}$ dimers are then transported to the nucleus where they stimulate

Abbreviations: HSC, hepatic stellate cells; $\alpha-S M A, \alpha$ smooth muscle actin; ECM, extracellular matrix; NFKB, nuclear factor $\kappa B$; IL-6, interleukin 6; ICAM-1, intercellular adhesion molecule 1 ; TNF- $\alpha$, tumour necrosis factor $\alpha$; TIMP-1, tissue inhibitor of metalloproteinase 1; MMP, matrix metalloproteinase; TGF, transforming growth factor; C/EBP, CCAAT/enhancer binding protein; bHLH, basic helix-loop-helix; M6P/IGFIIR, mannose 6-phosphate/ insulin-like growth factor II; PPAR $\gamma$, peroxisome proliferator activated receptor $\gamma ; l_{\kappa} B-\alpha$, inhibitor of $N F \kappa B$. 


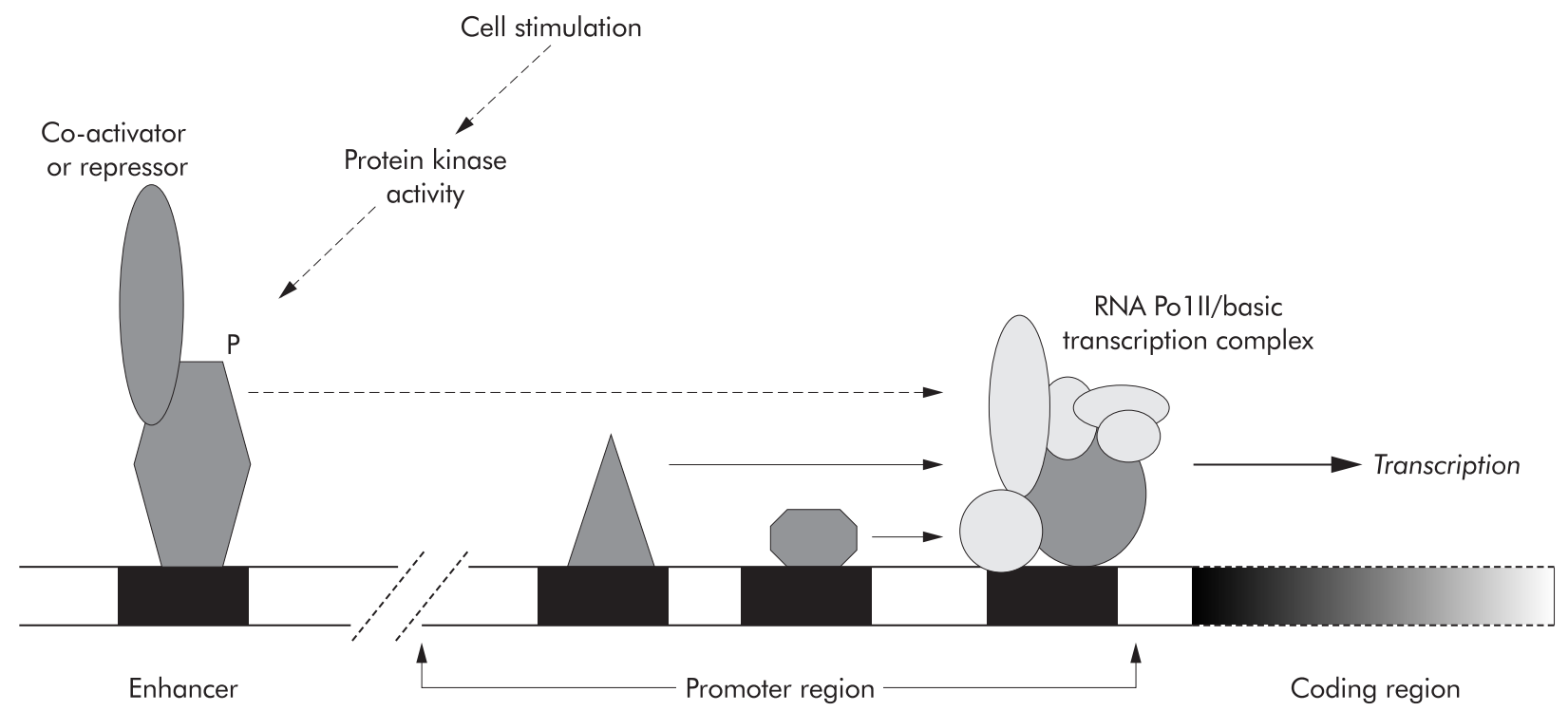

Figure 1 Events responsible for the transcriptional regulation of gene expression in mammalian cells. Transcription factors are depicted as gray shapes associating with the promoter and enhancer regions (black) of a gene and can stimulate or repress transcription by modulating the activity of the RNA polymerase II/basic transcription factor complex that locates to the start site of transcription. Enhancer elements can be separated (note break in the gene) by hundreds or thousands of base pairs from the promoter and can be found upstream or downstream of the transcription start site of the gene. The function of transcription factors can be attenuated by the action of coactivators or repressors and by post-translational modifications such as phosphorylation (P).

the transcription of genes that carry NFKB DNA binding motifs (GGGRNNYYCC, where $\mathrm{R}$ is purine, $\mathrm{Y}$ is pyrimidine, and $\mathrm{N}$ is any base), including the gene encoding I $\kappa \mathrm{B}-\alpha .{ }^{8}$ The ability of the IKB- $\alpha$ gene to enhance its rate of transcription in response to $\mathrm{NFKB}$ provides a negative feedback mechanism that within hours of the initial stimulatory event replenishes the cellular pool of the inhibitor and reverts NFKB back to its inactive state. ${ }^{8-10}$ Several studies have shown that HSC activation is associated with elevation of NFKB activity. ${ }^{11-14}$ Activated HSC predominantly express the classic p65:p50 complex but also express low levels of a p65 homodimer and high levels of a third and as yet undefined NFKB DNA binding complex. ${ }^{14}$

Induction of NFKB during HSC activation is distinct from the classical rapid NFKB response described above and is characterised by persistent elevation of $\mathrm{NF \kappa B}$ activity relative to that observed in quiescent HSC. ${ }^{14}$ As a result, activated HSC express a variety of NFKB responsive genes that are not associated with the quiescent HSC, including interleukin 6 (IL-6) and intercellular adhesion molecule 1 (ICAM-1). ${ }^{12-14}$ Precisely how the HSC regulates this shift towards a higher basal level of NFKB activity is not known but it is associated with a persistent reduction in the cytoplasmic and nuclear expression of I $\kappa-\alpha .{ }^{14}$ In addition, activated HSC express a hyperphosphorylated form of IкB- $\beta$ that can compete with IкB- $\alpha$ for interaction with NFKB but lacks the inhibitory properties of IKB- $\alpha$ and instead maintains $\mathrm{NFKB}$ in a transcriptionally active state. ${ }^{10}{ }^{14} \mathrm{NF \kappa B}$ activity can be further elevated in a classical transient manner following stimulation of HSC with cytokines, mitogens, and CD40 ligation, which result in enhanced expression of NFKB responsive genes such as ICAM-1, cyclooxygenase 2 , and interleukin $8 .{ }^{11-17}$

\section{"NFKB functions to promote the persistence of activated} HSC and a chronic wound healing response"

The role of NFKB in the activated HSC has been studied using pharmacological inhibitors, ${ }^{13}{ }^{14}$ or more specifically by

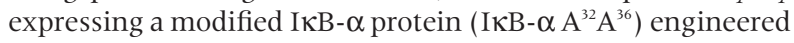
to be insensitive to the signalling pathways that serve to activate NFKB. ${ }^{13}{ }^{18}$ This latter strategy has ruled out a role for NFKB as a regulator of HSC activation (as assessed by morphology and expression of $\alpha$-SMA and $\alpha \mathrm{l}(\mathrm{I})$ collagen) and proliferation, but has demonstrated that NFKB protects HSC against tumour necrosis factor $\alpha$ (TNF- $\alpha)$ induced apoptosis. ${ }^{18}$ This important observation suggests that $\mathrm{NF \kappa B}$ functions to promote the persistence of activated HSC and a chronic wound healing response. In support of this idea, the NFKB inhibitor gliotoxin promotes accelerated recovery from carbon tetrachloride induced liver fibrosis in rats via its ability to stimulate HSC apoptosis. ${ }^{19}$

\section{AP-1}

AP-1 is composed of at least one Jun family protein (c-Jun, JunB, and JunD) which can either form a homodimer or heterodimerises with another Jun protein or with a member of the related Fos (c-Fos, FosB, $\Delta$ FosB, Fral, and Fra2) family. ${ }^{20}$ AP-1 dimers stimulate transcription by binding to a palindromic DNA sequence (TGAC/GTCA) commonly found in the promoter regions of a wide variety of genes involved in the control of cell growth, division, differentiation, and fate. ${ }^{2021}$ AP-1 activity is itself regulated by transcriptional and post-transcriptional control of the expression of the different fos and jun genes. ${ }^{21-23}$ However, AP-1 function is also controlled by its interaction with stimulatory or inhibitory proteins and through post-translational modifications of which phosphorylation of the Fos and Jun components by mitogen activated protein kinase and JNK signal transducers is the most important. ${ }^{23}{ }^{24}$ Quiescent HSC lack AP-1 activity but during culture activation transiently express c-Fos, Fral, c-Jun, and JunB which in the myofibroblast-like HSC are replaced by a persistent AP-1 activity consisting of Fra2, FosB, and JunD. ${ }^{25}$

"As Jun proteins are essential components of AP-1 dimers, JunD will therefore play a significant role in HSC activation and fibrosis"

Recent data from our laboratory have shown that JunD is functionally the most important of the constitutive AP- 1 factors in activated HSC, being required for induction of tissue inhibitor of metalloproteinase 1 (TIMP-1) and IL-6 gene transcription. ${ }^{26}$ Mutagenesis studies of the TIMP-1 and IL-6 
promoters showed that JunD must coregulate expression of these fibrogenic genes with other transcription factors expressed in activated HSC. In the case of the IL- 6 gene, the other critical factors are NFKB and NF-IL6 (CCAAT/enhancer binding protein $\beta(\mathrm{C} / \mathrm{EBP} \beta)$ ) together with an as yet undefined heterodimeric partner for JunD. ${ }^{26}$ By contrast, TIMP-1 gene transcription is regulated by JunD homodimers which appear to operate in concert with a $30 \mathrm{kDa}$ nuclear protein that is induced in culture activated HSC and binds to a novel and essential DNA binding site in the TIMP-1 promoter called UTE- $1 .{ }^{27}$ JunD is also the predominant Jun family protein expressed in HSC isolated from the liver of carbon tetrachloride injured rats, ${ }^{26}$ and as Jun proteins are essential components of AP-1 dimers, JunD will therefore play a significant role in HSC activation and fibrosis.

The constitutive AP- 1 activity of activated HSC can be further stimulated and may also be modified in terms of expression of specific Fos and Jun factors by acetaldehyde, aldehydic end products, cytokines, and fibronectin. ${ }^{28-30}$ Most significantly, these agents can promote expression and activation (via stimulation of mitogen activated protein kinase and JNK) of c-Jun homodimers and/or c-Jun:Fos heterodimers, which have been implicated as transcriptional regulators of the collagenase (matrix metalloproteinases (MMP)1/13) and stromelysin (MMP3) genes. ${ }^{31-33}$ Therefore, shifts in the fine balance of the expression and activity of the different forms of AP- 1 and their target MMP and TIMP genes in response to cues from the microenvironment of the activated HSC will promote the ability of the cell to remodel the hepatic ECM.

\section{KRUPPEL-LIKE TRANSCRIPTION FACTORS}

GC rich sequences and related GT or CACCC boxes are found in the regulatory regions of many profibrogenic genes expressed in activated HSC, including the $\alpha \mathrm{l}(\mathrm{I})$ collagen, transforming growth factor (TGF)- $\beta 1$ and type I and II TGF- $\beta$ receptor genes. These sequences are recognition sites for the Kruppel-like transcription factor family that have the common structural feature of three Kruppel-like zinc fingers that mediate their specific DNA binding properties. This large and expanding family of transcription factors characteristically regulates expression of ubiquitously expressed or housekeeping genes but also control the transcription of tissue specific genes. ${ }^{34}$

\section{"Transcriptional regulators of HSC activation are in turn under the control of other transcription factors"}

Activated HSC express at least three members of the Kruppel-like transcription factor family, SP1, BTEB1, and KLF6 (formerly Zf9), all of which have the ability to regulate transcription of the $\alpha \mathrm{l}(\mathrm{I})$ collagen gene. ${ }^{30}{ }^{35} 36$ SPI DNA binding activity and protein expression are induced during HSC activation by an undefined post-transcriptional mechanism. ${ }^{35}$ SPl is a potent transcriptional regulator of $\alpha \mathrm{l}(\mathrm{I})$ collagen expression and it associates with two GC rich regions (FPl and FP2) of the $\alpha 1(\mathrm{I})$ collagen gene promoter in activated HSC. $^{1635}$ Mutagenesis of FP1 and FP2 results in greatly reduced activity of the $\alpha \mathrm{l}(\mathrm{I})$ collagen gene promoter in HSC and overexpression of SPI enhances promoter activity ${ }^{16}$ Rippe and colleagues demonstrated that NFKB is a potent repressor of $\alpha \mathrm{l}$ (I) collagen gene transcription and operates via a mechanism that appears to involve formation of direct interactions with SPl. ${ }^{16}$ Hence expression of $\alpha \mathrm{l}(\mathrm{I})$ collagen in activated HSC may be attenuated by differential activation of SPI and $\mathrm{NFKB}$ in response to cellular stimulation by growth factors and cytokines. Of relevance, the inflammatory cytokine TNF- $\alpha$ induces NFKB activity in activated HSC and also inhibits $\alpha \mathrm{I}$ (I) collagen gene expression ${ }^{16}{ }^{37}$ BTEBI mediates UV and acetaldehyde induced activation of $\alpha \mathrm{l}$ (I) collagen gene transcription via a GC box that is distinct from the FP1 and FP2 sites and is located in a region $(-1484$ to -1476$)$ that is far removed upstream of the transcription start site of the gene. ${ }^{30}{ }^{38}$ The BTEBl gene is a target for AP-1 and induction of BTEBl expression in acetaldehyde treated HSC is mediated via activation of JNK and elevation of AP-1 activity. ${ }^{30}$ Hence expression of transcriptional regulators of HSC activation are in turn under the control of other transcription factors. It is expected that by unravelling these complex networks of gene regulation it will eventually be possible to identify transcription factors that act as master regulators or orchestrators of HSC activation.

One potential master regulator of HSC activation is the recently discovered Kruppel-like factor KLF6. In vivo and in vitro studies have shown that induction of KLF6 gene transcription is a very early event in HSC activation and that expression of KLF6 is a persistent feature of the fully activated myofibroblastic HSC. ${ }^{36}$ In addition to stimulating transcription of the $\alpha \mathrm{l}$ (I) collagen gene, ${ }^{36}$ KLF6 also functions as a regulator of several key components of the fibrogenic TGF- $\beta 1$ pathway. ${ }^{39}{ }^{40}$ Activated HSC not only secrete TGF- $\beta 1$ but also express type I and II TGF- $\beta$ receptors and the urokinase-type plasminogen activator which activates latent TGF- $\beta 1{ }^{41}{ }^{42}$ The genes encoding TGF- $\beta 1$, its type I and II receptors, and urokinase-type plasminogen activator are all under the transcriptional control of GC box motifs that are targets for KLF6 activity ${ }^{39}{ }^{40}$ Future studies in KLF6 gene knockout mice should soon confirm a major regulatory role for this protein in hepatic fibrogenesis.

\section{C/EBP}

Members of the CCAAT/enhancer binding protein (C/EBP) family of transcription factors have also been implicated as regulators of $\alpha \mathrm{l}(\mathrm{I})$ collagen gene expression. These proteins consist of a conserved DNA binding region and a leucine zipper dimerisation domain; the more divergent transactivation domain responsible for transcriptional activation and/or repression is found at the $\mathrm{N}$ terminal end of the proteins. ${ }^{43}$ Work by the group led by Rojkind has shown that both TGF- $\beta$ and acetaldehyde can increase expression of the $\alpha \mathrm{l}(\mathrm{I})$ collagen gene in HSC by promoting the formation of $\mathrm{H}_{2} \mathrm{O}_{2}{ }^{44}{ }^{45}$ Accumulation of intracellular $\mathrm{H}_{2} \mathrm{O}_{2}$ appears to stimulate expression of a p35 isoform of C/EBP $\beta$ and promotes the binding of this factor to a TGF- $\beta$ responsive element located between nucleotides -378 to -345 in the mouse $\alpha \mathrm{l}$ (I) collagen gene promoter. ${ }^{44}$ Interaction of p35 C/EBP $\beta$ with this element then increases the rate of transcription of the $\alpha \mathrm{l}$ (I) collagen gene. Rojkind's group went on to show that TNF- $\alpha$ induced downregulation of $\alpha \mathrm{l}$ (I) collagen gene transcription also involves interaction of C/EBP factors with the -378 to -345 region of the promoter. ${ }^{46}$ TNF- $\alpha$ stimulates binding of a p20 isoform of C/EBP $\beta$ and $\mathrm{C} / \mathrm{EBP} \delta$, both of which act as repressors of $\alpha \mathrm{l}(\mathrm{I})$ collagen gene transcription. ${ }^{44}$ The TNF- $\alpha$ induced shift in C/EBP $\beta$ isoform binding to the promoter, from the p35 to the p20 isoform, is significant as the p20 C/EBP $\beta$ lacks a transactivation domain and therefore functions as a transcriptional repressor. ${ }^{47}$

\section{E-bOX TRANSCRIPTION FACTORS AND c-Myb}

E-box elements consist of a CANNTG (where $\mathrm{N}$ is any base) motif and bind members of the basic helix-loop-helix (bHLH) transcription factor family. ${ }^{48}$ bHLH proteins can be broadly placed into two categories: class A factors that are expressed in a ubiquitous pattern and class $\mathrm{B}$ factors that are expressed in a tissue restricted manner. bHLH factors function as dimers that can include combinations of both class A and class B proteins; this creates a diverse range of transcription factors that are involved in regulating cell growth and differentiation pathways. ${ }^{49-51}$ 


\begin{abstract}
"It is important to determine the role played by MyoD as a transcriptional regulator of the myogenic properties of HSC"
\end{abstract}

The myogenic transcription factor MyoD is a class B bHLH protein that has a highly restricted pattern of expression limited to cells of the skeletal muscle lineage, myoid cells of the thymus, and myofibroblasts. ${ }^{52}$ MyoD functions in cooperation with its related myogenic transcription factors Myf5, myogenin, MRF4, and the MEF2 group to regulate skeletal muscle cell determination and differentiation. ${ }^{49}$ The recent observation that MyoD is also expressed by activated human and rat HSC in a biologically active form indicates that it may also regulate the myogenic and contractile properties of the myofibroblast-like HSC. ${ }^{52-54}$ Although activated HSC are often referred to as being smooth muscle myofibroblast-like on the basis of their expression of $\alpha$-SMA, they additionally acquire expression of the contractile IIa, IId, and perinatal skeletal muscle myosin heavy chain isoforms. ${ }^{5253}$ As the contractile nature of the activated HSC contributes to portal hypertension, an important pathological feature of the cirrhotic liver, ${ }^{55}$ it is important to determine the role played by MyoD as a transcriptional regulator of the myogenic properties of HSC. As forced expression of MyoD can trigger the terminal differentiation of fibroblasts into skeletal muscle cells, ${ }^{56}$ it will also be of great interest to determine how activated HSC can exist in a state in which they exhibit phenotypic features of both fibroblasts and muscle cells.

E-box factors have been implicated as transcriptional regulators of the mannose 6-phosphate/insulin-like growth factor II (M6P/IGFIIR) gene, which has been postulated to play a key role in fibrogenesis by binding latent TGF- $\beta$ at the cell surface, thereby promoting its activation. ${ }^{57}{ }^{58}$ Weiner $e t$ al have described three functional E-box elements in the M6P/IGFIIR gene. Two of these elements are located proximal to the transcription start site and bind a novel $75 \mathrm{kDa}$ (P75) nuclear protein that is downregulated in activated HSC. ${ }^{57}$ As mutations of these proximal E-box elements enhance promoter activity, it is thought that P75 is a repressor of transcription. The third E-box is located distal to the transcription start site, is essential for M6P/ IGFIIR promoter activity, and displays enhanced protein binding in HSC treated with platelet derived growth factor $\mathrm{BB}^{58}$ The as yet uncharacterised factor binding to this distal E-box is distinct from the P75 repressor. These data suggest that activation of latent TGF- $\beta$, a key step in fibrogenesis, is influenced by the activity of repressor and activator bHLH factors.

A major myogenic feature of activated HSC is their abundant expression of $\alpha$-SMA; until recently there was little information available regarding the control of $\alpha$-SMA gene transcription in HSC. However, Buck et al have now demonstrated that a region of 271 base pairs located immediately upstream of the transcription start site of the rat $\alpha$-SMA gene is sufficient to direct transcription in activated rat HSC. ${ }^{59}$ This region contains two E-boxes of which the E-box located proximal to the start site of transcription has previously been implicated as a binding site for the transcription factors C-Myb and NFKB, both of which are induced during HSC activation. ${ }^{11} \mathrm{C}-\mathrm{Myb}$ is the prototype of a family of related Myb proteins that act as either positive or negative transcriptional regulators of a wide variety of genes. ${ }^{60}$ Buck et al showed that c-Myb binds to an E-box-like sequence that is located immediately adjacent to the proximal E-box. ${ }^{59}$ The most exciting aspect of this study was the demonstration that c-Myb not only stimulates the activity of a transfected $\alpha$-SMA promoter but is also required for expression of the endogenous $\alpha$-SMA gene. Perhaps even more remarkable was the finding that disruption of c-Myb expression using antisense oligonucleotides resulted in a blockade of HSC activation, as judged by morphological criteria. Further studies on c-Myb function in the activated HSC, including confirmation of an in vivo role in HSC activation and fibrosis, are now required. But this study exemplifies how an understanding of the transcriptional regulation of HSC activation may identify potential therapeutic targets.

\section{PPAR $\gamma$ AND TRANSCRIPTION FACTOR ACTIVITIES ASSOCIATED WITH THE QUIESCENT HSC}

The transcription factors discussed so far have the common feature of being associated with the activated phenotype of HSC. It is however also important to study the function of transcription factors that are active in quiescent HSC, especially if these activities are diminished during HSC activation. These latter types of transcription factors may function as inhibitors of HSC activation, as suggested by a series of recent papers describing the peroxisome proliferator activated receptor $\gamma$ (PPAR $\gamma$ ) as a novel modulator of HSC biology. ${ }^{61-63}$

PPAR $\gamma$ is a member of the steroid/thyroid hormone nuclear receptor superfamily that is implicated in metabolic diseases such as diabetes and operates as an obligate heterodimer with the retinoid X receptor to regulate gene transcription and cellular differentiation. ${ }^{64}{ }^{65}$ Expression of PPAR $\gamma$ protein is dramatically reduced in HSC activated both in vitro (human and rat cells) and in vivo (rat cells isolated from bile duct ligated rats ). ${ }^{61-63}$ These studies also showed that HSC activation is associated with significantly reduced activity of transfected synthetic promoters that contain multiple copies of the PPAR response element. ${ }^{62}{ }^{63}$

"There may be genuine promise in the clinical use of PPAR $\gamma$ ligands for the treatment of hepatic fibrosis and cirrhosis"

A variety of PPAR $\gamma$ agonists and antagonists are available that act as ligands for the nuclear receptor and either promote or inhibit its transcriptional activity. ${ }^{65}$ The PPAR $\gamma$ agonist 15-deoxy $\Delta^{12,14}$-prostaglandin $\mathrm{J}_{2}$ was shown in all three of the reported studies to block multiple phenotypic features of the activated HSC including platelet derived growth factor induced proliferation, DNA synthesis, chemotaxis, and expression of $\alpha$-SMA, $\alpha \mathrm{l}(\mathrm{I})$ collagen, and monocyte chemotactic protein $1{ }^{61-63}$ Impressively, these effects were reproduced when exposing activated HSC to a variety of different PPAR $\gamma$ agonists and were abrogated in HSC treated with a PPAR $\gamma$ antagonist. If these exciting observations can be reproduced in animal models of hepatic fibrosis then there may be genuine promise in the clinical use of PPAR $\gamma$ ligands for the treatment of hepatic fibrosis and cirrhosis.

Reports of other transcription factors that undergo diminution of their expression or activity as HSC activate are rare but two such factors have been identified that belong to the bHLH family. One is the P75 repressor of M6P/IGFIIR gene transcription and the other is Id $1 .{ }^{54}$ Quiescent HSC express abundant levels of Id 1 mRNA that are rapidly and persistently diminished on culture induced activation. Id l is a bHLH factor that lacks a DNA binding domain but can form transcriptionally inactive dimers with other bHLH transcription factors. ${ }^{66}$ When Id $\mathrm{l}$ is forcibly overexpressed it will block the terminal differentiation of muscle and nerve cells by preventing the function of myogenic and neurogenic bHLH proteins. As induction of MyoD DNA binding activity coincides with loss of Id l expression in HSC, it is plausible that Idl acts to inhibit the activity of MyoD in quiescent HSC. ${ }^{54}$ Finally, Bahr and colleagues $^{25}$ and Knittel and colleagues ${ }^{67}$ have also identified Ets-1, a member of the Ets transcription factor family that regulates expression of many genes involved in ECM synthesis and turnover, including TIMP- $1,{ }^{25}{ }^{68}$ as a factor that undergoes downregulation of its expression during HSC activation. The functional significance of P75, Id l, and Ets- 1 as regulators of the HSC phenotype should now be determined using viral 
or plasmid based gene transfer systems to generate forced overexpression of these factors in the activated HSC.

\section{SUMMARY AND DIRECTIONS FOR FUTURE RESEARCH}

The complexity of events at the level of gene transcription associated with HSC activation appears daunting at first. In particular, the requirement for the coordinated activity of several transcription factors to control expression of any one fibrogenic protein such as $\alpha 1$ (I) collagen (SP1, KLF6, BTEB, AP- 1, NFKB, C/EBP, PPAR $\gamma$ ) may seem discouraging. However, by identifying transcription factors such as JunD, KLF-6, c-Myb, and PPAR $\gamma$ that are individually able to contribute to the transcriptional regulation of multiple phenotypic characteristics of the activated HSC, it will eventually be possible to map the major transcriptional pathways that result in HSC activation and thereby promote fibrosis. Future efforts should be particularly directed to in vivo studies with gene deleted mice or virus vector mediated delivery of dominant negative inhibitors to confirm a pathophysiological role for these potential orchestrators of HSC activation in the fibrogenic process. There should also be attention placed on the regulation of gene transcription at the level of the chromatin structure which, through the tightly regulated activity of the histone acetyl transferases and the histone deacetylases, can exert global control over gene expression. ${ }^{4669}$ The findings of these future studies will provide mechanisms for the experimental manipulation of the HSC phenotype and are likely to lead to the discovery and development of new therapies in the treatment of hepatic fibrosis.

\section{ACKNOWLEDGEMENTS}

Current research grant support was provided to DAM by the MRC (G9900951 and G9900297), the Wellcome Trust (050443/Z), and the British Liver Trust. DES is grateful to Hope (formerly the Wessex Medical Trust) for a PhD studentship award. The authors wish to thank Professor John Iredale for critical reading and advice during the preparation of the manuscript.

\section{Authors' affiliations}

D A Mann, D E Smart, Liver Group, Division of Infection, Inflammation, and Repair, University of Southampton, Southampton, UK

\section{REFERENCES}

1 Friedman SL. The cellular basis of hepatic fibrosis. N Engl J Med 1993:328: 1828-35.

2 Friedman SL. Molecular regulation of hepatic fibrosis, an integrated cellular response to tissue injury. J Biol Chem 2000;275:2247-50.

3 Arthur MJ, Mann DA, Iredale JP. Tissue inhibitors of metalloproteinases, hepatic stellate cells and liver fibrosis. J Gastroenterol Hepatol 1998;13(suppl):S33-8.

4 Lemmon B, Tijan R. Orchestrated response: a symphony of transcription factors for gene control. Genes Dev 2000;14:2551-69.

5 Calkhoven CF, Ab G. Multiple steps in the regulation of transcription factor level and activity. Biochem J 1996;317:329-42.

6 Kouzarides T. Acetylation: a regulatory modification to rival phosphorylation. EMBO J 2000;19:1176-9.

7 Desterro JM, Rodriguez MS, Hay RT. Regulation of transcription factors by protein degradation. Cell Mol Life Sci 2000;57:1207-19.

8 Baldwin AS. The NF- $\mathrm{KB}$ and $\mathrm{l} \mathrm{KB}$ proteins: new discoveries and insights. Annu Rev Immunol 1996;14:649-81.

9 Ghosh S, May M, Kopp EB. NF-kB and Rel proteins: evolutionary conserved mediators of immune responses. Annu Rev Immunol 1998; 16:225-60.

10 Karin M, Ben-Neriah Y. Phosphorylation meets ubiquitination: The control of NF-KB activity. Annu Rev Immunol 2000;18:621-63.

11 Lee KS, Buck M, Houglum K, et al. Activation of hepatic stellate cells by TGF alpha and collagen type $I$ is mediated by oxidative stress through c-myb expression. J Clin Invest 1995;96:2461-8.

12 Hellerbrand C, Jobin C, Licato LL, et al. Cytokines induce NF-KB in activated but not in quiescent rat hepatic stellate cells. Am J Physiol 1998;275:G269-78.

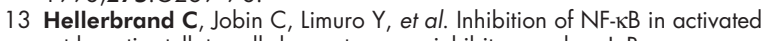
rat hepatic stellate cells by proteasome inhibitors and an $I_{\kappa} B$ super-repressor. Hepatology 1998;27:1285-95.

14 Elsharkawy AM, Wright MC, Hay RT, et al. Persistent activation of nuclear factor-kappa $B$ in cultured rat hepatic stellate cells involves the induction of potentially novel Rel-like factors and prolonged changes in the expression of IKB family proteins. Hepatology 1999;30:761-9.

15 Gallois C, Habib A, Tao J, et al. Role of NF-KB in the antiproliferative effect of endothelin- 1 and tumor necrosis factor- $\alpha$ in human hepatic stellate cells. J Biol Chem 1998;273:23183-90.

16 Rippe RA, Schrum LW, Stefanovic B, et al. NF-KB inhibits expression of the $\alpha 1$ (I) collagen gene. DNA Cell Biol 1999;18:751-61

17 Scwabe RF, Schnabl B, Kweon YO, et al. CD40 activated NF-KB and c-Jun $\mathrm{N}$-terminal kinase and enhances chemokine secretion on activated human hepatic stellate cells. J Immunol 2001;166:6821-9.

18 Lang A, Schoonhovan R, Tuvia S, et al. Nuclear factor kappaB in proliferation, activation and apoptosis in rat hepatic stellate cells. J Hepatol 2000;33:49-58.

19 Wright MC, Issa R, Smart DE, et al. Stimulated apoptosis of hepatic stellate cells enhances the resolution of liver fibrosis. Gastroenterology 2001:121:685-98.

20 Vogt PK. Jun, the oncoprotein. Oncogene 200 1;20:2365-77.

21 Shaulian E, Karin M. AP-1 in cell proliferation and survival. Oncogene $2001 ; 20: 2390-400$.

22 Mechta-Grigoriou F, Gerald D, Yaniv M. The mammalian Jun proteins: redundancy and specificity. Oncogene 2001;20:2378-89.

23 Karin M, Liu Z, Zandi E. AP-1 function and regulation. Curr Opin Cell Biol 1997;9:240-6

24 Chinenov $Y$, Kerppola TK. Close encounters of many kinds: Fos-Jun interactions that mediate transcription regulatory specificity. Oncogene $2001 ; 20: 2438-52$

25 Bahr MJ, Vincent KJ, Arthur MP, et al. Control of the tissue inhibitor of metalloproteinases-1 promoter in culture-activated rat hepatic stellate cells: regulation by activator protein-1 DNA binding proteins. Hepatology 1999;29:839-48.

26 Smart DE, Vincent KJ, Arthur MP, et al. JunD regulates transcription of the tissue inhibitor of metalloproteinases- 1 and interleukin- 6 genes in activated hepatic stellate cells. J Biol Chem 2001;276:24414-21.

27 Trim JE, Samra SK, Arthur MP, et al. Upstream tissue inhibitor of metalloproteinases-1 (TIMP-1) element-1, a novel and essential regulatory DNA motif in the human TIMP-1 gene promoter, directly interacts with a 30-kDa nuclear protein. J Biol Chem 2000;275:6657-63.

28 Poulos JE, Weber JD, Bellezzo AM, et al. Fibronectin and cytokines increase JNK, ERK, AP-1 activity and transin gene expression in rat hepatic stellate cells. Am J Physiol 1997;273:G804-11.

29 Parola M, Robino G, Marra F, et al. HNE interacts directly with JNK isoforms in human hepatic stellate cells. J Clin Invest 1998;102:194250.

30 Chen A, Davis BH. The DNA binding protein BTEB mediates acetaldehyde-induced Jun $\mathrm{N}$-terminal kinase-dependent $\alpha 1$ (I) collagen gene expression in rat hepatic stellate cells. Mol Cell Biol 2000;20:2818-26.

31 Buttice G, Quinones S, Kurkinen M. The AP-1 site is required for basal expression but is not necessary for TPA-response of the human stromelysin gene. Nucleic Acids Res 1991;19:3723-31.

32 Newberry EP, Willis D, Latifi T. Fibroblast growth factor receptor signaling activates the human interstitial collagenase promoter via the bipartite Ets-AP-1 element. Mol Endocrinol 1997;11:11129-44.

33 Solis-Herruzo, Rippe RA, Schrum LW, et al. Interleukin-6 increases rat metalloproteinase-13 gene expression through stimulation of activator protein 1 transcription in cultured fibroblasts. J Biol Chem 1999;274:30919-26.

34 Turner J, Crossley M. Mammalian Krüppel-like transcription factors: more than just a pretty finger. Trends Biochem 1999;24:236-40.

35 Rippe RA, Almounajed G, Brenner DA. Spl binding activity increases in activated lto cells. Hepatology 1995;22:241-51.

36 Ratziu V, Lalazar A, Wong L, et al. Zf9, a Kruppel-like transcription factor up-regulated in vivo during early hepatic fibrosis. Proc Natl Acad Sci USA 1998;95:9500-5.

37 Armendariz-Borunda J, Katayama K, Seyer JM. Transcriptional mechanisms of type I collagen gene expression are differentially regulated by interleukin-1 beta, tumor necrosis factor alpha and transforming growth factor beta in Ito cells. J Biol Chem 1992;267:14316-21

38 Chen A, Davis BH. UV irradiation activates JNK and increases $\alpha$ l(I) collagen gene expression in rat hepatic stellate cells. J Biol Chem 1999;274:158-64.

39 Kim Y, Ratziu V, Cho S-G, et al. Transcriptional activation of transforming growth factor $\beta 1$ and its receptors by the Kruppel-like factor Zf9/core promoter-binding protein and Spl. J Biol Chem 1998;273:33750-8.

40 Kojima S, Hayashi S, Shimokado K, et al. Transcriptional activation of urokinase by the Kruppel-like factor ZF9/COPEB activated latent TGF-beta 1 in vascular endothelial cells. Blood 2000;15:1309-16.

41 Friedman SL, Yamasaki G, Wong L. Modulation of transforming growth factor beta receptors of rat lipocytes during the hepatic wound healing response. Enhanced binding and reduced gene expression accompany cellular activation in culture. J Biol Chem 1994;269:10551-18.

42 Leyland $\mathbf{H}$, Gentry J, Arthur MP, et al. The plasminogen-activating system in hepatic stellate cells. Hepatology 1996;24:1172-8.

43 Lekstrom-Himes J, Xanthopoulos KG. Biological role of the CCAAT/enhancer-binding protein family of transcription factors. J Biol Chem 1998;273:28545-8.

44 Garcia-Trevijano ER, Iraburu M, Fontana L, et al. Transforming growth factor $\beta 1$ induces the expression of $\alpha$ l (I) procollagen mRNA by a hydrogen peroxide-C/EBP $\beta$-dependent mechanism in rat hepatic stellate cells. Hepatology 1999;29:960-70. 
45 Greenwel P, Dominguez-Rosales J-A, Mavi G et al. Hydrogen peroxide: a link between acetaldehyde-elicited $\alpha 1$ (I) collagen gene up-regulation and oxidative stress in mouse hepatic stellate cells. Hepatology 2000;31:109-16.

46 Iraburu MJ, Dominguez-Rosales J-A, Fontana L, et al. Tumor necrosis factor $\alpha$ down-regulates expression of the $\alpha 1$ (I) collagen gene in rat hepatic stellate cells through a p20C/EBP $\beta$ - and C/EBPS-dependent mechanism. Hepatology 2000;31:1086-93.

47 Descombes $\mathbf{P}$, Shibler U. A liver-enriched transcriptional activator protein, LAP, and a transcriptional inhibitory protein, LIP, are translated from the same mRNA. Cell 1991;67:569-76.

48 Blackwell TK, Weintraub H. Differences and similarities in DNA-binding preferences of MyoD and E2A protein complexes revealed by binding site selection. Science 1990;250:1104-10.

49 Yun K, Wold B. Skeletal muscle determination and differentiation: story of a core regulatory network and its context. Curr Opin Cell Biol 1996;8:877-89

50 Fisher A, Caudy M. The function of hairy-related bHLH repressor proteins in cell fate decisions. Bioessays 1998;20:298-306.

51 Guillemot F. Vertebrate bHLH genes and the determination of neuronal fates. Exp Cell Res 1999;253:357-64.

52 Walker GA, Guerrero IA, Leinwand LA. Myofibroblasts: molecular crossdressers. Curr Top Dev Biol 2001;51:91-107.

53 Mayer DC, Leinwand LA. Sarcomeric gene expression and contractility in myofibroblasts. J Cell Biol 1997;139:1477-84.

54 Vincent KJ, Jones E, Arthur MP, et al. Regulation of E-box DNA binding during in vivo and in vitro activation of rat and human hepatic stellate cells. Gut 2001;49:713-19

55 Bosch J, Garcia-Pagan JC. Complications of cirrhosis. I. Portal hypertension. J Hepatol 2000;32(suppl 1):141-56.

56 Davis RL, Weintraub $H$, Lassar $A B$. Expression of a single transfected cDNA converts fibroblasts into myoblasts. Cell 1987;51:987-1000.

57 Weiner JA, Chen A, Davis BH. E-box repressor is down-regulated in hepatic stellate cells during up-regulation of mannose-6-phosphate/ insulin-like growth factor II receptor expression in early hepatic fibrogenesis. J Biol Chem 1998;273:15913-19.
58 Weiner JA, Chen A, Davis BH. Platelet-derived growth factor is a principal inductive factor modulating mannose 6-phosphate/insulin-like growth factor receptor gene expression via a distal E-box in activated hepatic stellate cells. Biochem J 2000;345:225-31.

59 Buck M, Kim DJ, Houglum K, et al. c-Myb modulates transcription of the alpha-smooth muscle actin gene in activated hepatic stellate cells. Am J Physiol 2000;278:G321-8.

60 Weston K. Myb proteins in life, death and differentiation. Curr Opin Genet Dev 1998:8:76-81.

61 Miyahara T, Schrum L, Rippe R, et al. Peroxisome proliferator-activated receptors and hepatic stellate cell activation. J Biol Chem 2000;275:35715-22.

62 Galli A, Crabb D, Price D, et al. Peroxisome proliferator-activated receptor $\gamma$ transcriptional regulation is involved in platelet-derived growth factor-induced proliferation of human hepatic stellate cells. Hepatology 2000;31:101-8.

63 Marra F, Efsen A, Romanelli RG, et al. Ligands of peroxisome proliferator-activated receptor $\gamma$ modulate profibrogenic and proinflammatory actions in hepatic stellate cells. Gastroenterology 2000;1 19:466-78.

64 Chinetti G, Fruchart JC, Staals B. Peroxisome proliferator-activated receptors (PPARs): nuclear receptors at the crossroads between lipid metabolism and inflammation. Inflamm Res 2000;49:497-505.

65 Wilson TM, Lambert MH, Kliewer SA. Peroxisome proliferator-activated receptor $\gamma$ and metabolic disease. Ann Rev Biochem 2001;70:341-67.

66 Norton JD. ID helix-loop-helix proteins in cell growth, differentiation and tumorigenesis. J Cell Sci 2000;1 13:3897-905.

67 Knittel T, Kobold D, Dudas J, et al. Role of the Ets-1 transcription factor during activation of rat hepatic stellate cells in culture. Am J Pathol 1999:155: 1841-8.

68 Trojanawska $M$. Ets factors and regulation of extracellular matrix. Oncogene 2000;19:6464-71

69 Niki T, Rombouts K, De Blesser P, et al. A histone deacetylase inhibitor, trichostatin A, suppresses myofibroblastic differentiation of rat hepatic stellate cells in primary culture. Hepatology 1999;29:858-67.

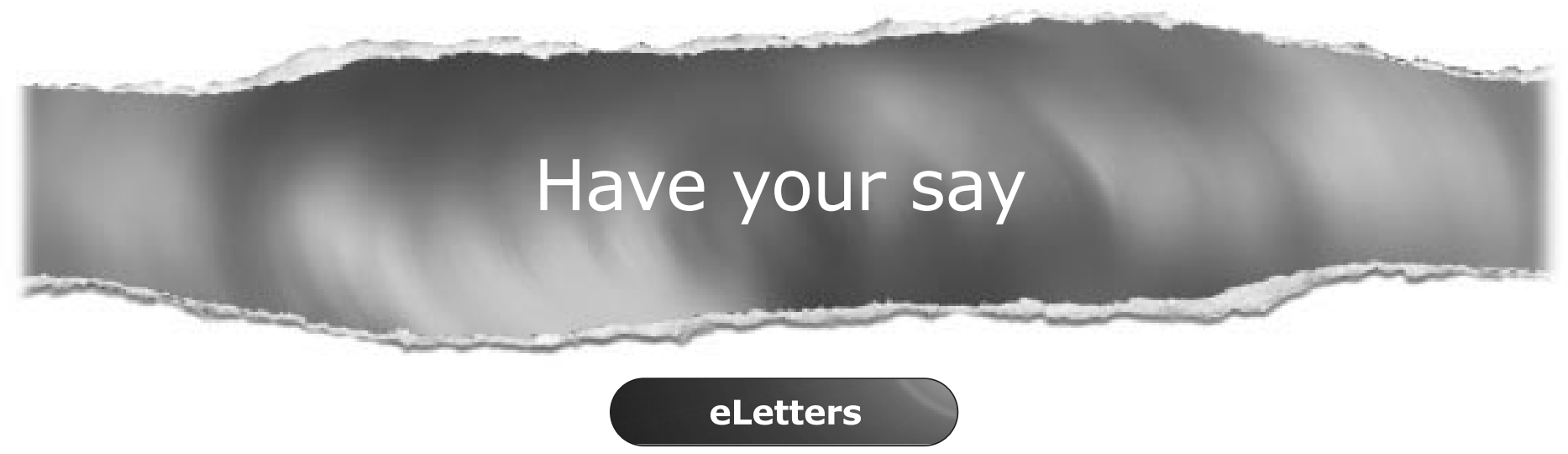

If you wish to comment on any article published in Gut you can send an eLetter using the eLetters link at the beginning of each article. Your response will be posted on Gut online within a few days of receipt (subject to editorial screening). 